\title{
Distribución potencial de Sanionia spp. en dos momentos del Holoceno en península Fildes isla Rey Jorge Antártica
}

\author{
Potential distribution of Sanionia spp. at two moments of the \\ Holocene in Fildes Peninsula King George Island Antarctica
}

Inti González ${ }^{1}$, Ingrid Hebel ${ }^{2}$, Ricardo Jaña ${ }^{3}$

\section{Resumen}

Se genera el primer modelo de distribución potencial para los musgos Sanionia spp. en península Fildes, Shetland del Sur, Antártica, utilizando el algoritmo de máxima entropía (Maxent) para dos momentos del Holoceno. El modelo de distribución potencial de Sanionia spp. calibrado para el momento temporal presente (MDP S. Presente) se ejecuta para períodos de tiempo pasados $11 \mathrm{ka}$ (MDP S. ${ }^{11 \mathrm{ka}) \text { y } 6}$ ka (MDP S. ${ }^{6 \mathrm{ka}}$ ). Conociendo que el nicho ecológico es un set de condiciones bióticas y abióticas en el cual la especie es capaz de mantenerse estable en tamaño poblacional, se utilizaron para la calibración del MDP S. Presente variables abióticas como la topografía y temperatura. Las variables topográficas son elevación, pendiente y exposición, construidas en base a un modelo de elevación digital del área libre de hielo de península Fildes. Para los MDP S. ${ }^{11}$ ka y MDP S. ${ }^{6}$ ka , se construyen las variables con los datos del modelo de elevación digital actual modificado según la reconstrucción de posición de la capa de hielo, el nivel del mar y valores de anomalías de temperatura obtenidos del testigo de hielo de la isla James Ross. El modelo de distribución potencial define que la probabilidad de presencia en península Fildes para el momento actual es de 29,5\%, para el momento 6 ka la probabilidad de presencia es de $58,9 \%$ y para el momento 11 ka la probabilidad es de 69,9\%. La probabilidad de presencia de Sanionia spp. en península Fildes ocurre con temperaturas entre los $-2,4^{\circ} \mathrm{C}$ y los $-3,0^{\circ} \mathrm{C}$, alturas bajo los $130 \mathrm{~m}$, pendientes bajo el $52 \%$ y exposiciones sur - este, sur- oeste y noroeste.

\section{Palabras Clave:}

Modelo distribución potencial, Maxent, Sanionia spp., áreas libres de hielo, península Fildes, Antártica.

\section{Abstract}

The first potential distribution model for mosses, Sanionia spp., in Fildes peninsula, South Shetland, Antarctica was generated using the maximum entropy algorithm (Maxent) for two Holocene moments. The potential distribution model of Sanionia spp. calibrated for the present time (DPM $S$. Present) was executed for past periods, $11 \mathrm{ka}$ (DPM S. ${ }^{11 \mathrm{ka})}$ and $6 \mathrm{ka}$ (DPM $S$. $6 \mathrm{ka})$. Knowing that the ecological niche is a set of biotic and abiotic conditions where the species is able to remain stable in population size, abiotic variables, such as topography and temperature were used for the calibration of the DPM S. Present. The topographic variables are elevation, slope and exposure, constructed based on a digital elevation model of the ice-free area of the Fildes peninsula. For the DPM S. ${ }^{11} \mathrm{ka}$ and DPM S. ${ }^{6 \mathrm{ka}}$ the variables are constructed with the data of the

Cambio Climático y Variaciones Recientes,

Centro Regional Fundación CEQUA, Punta Arenas, Chile.

$>$ into.gonzalez@cequa.cl

2 Laboratorio de Biotecnología Vegetal, Departamento de Agropecuaria y Acuicultura, Universidad de Magallanes, Punta Arenas, Chile.

Departamento Científico, Instituto Antártico Chileno, Punta Arenas, Chile. 
current digital elevation model modified according to the reconstruction of ice sheet position, sea level and temperature anomaly values obtained from the ice core on James Ross island. The model established that the probability of presence in the Fildes peninsula for the current moment is $29.5 \%$, for the moment $6 \mathrm{k}$ the probability of presence is $58.9 \%$ and for the moment 11 ka the probability is $69.9 \%$. The probability of presence of Sanionia spp. in Fildes peninsula occurs with temperatures between $-2.4^{\circ} \mathrm{C}$ and $-3.0^{\circ} \mathrm{C}$, heights below $130 \mathrm{~m}$, slopes below $52 \%$ and in south - east, south - west and northwest aspect.

\section{Key words:}

Potencial distribution model, Maxent, Sanionia spp, ice free areas, Fildes peninsula, Antarctica.

\section{INTRODUCCIÓN}

La evidencia de registros paleontológicos y geomorfológicos indica que la máxima extensión de la capa de hielo de península Antártica alcanzó el borde de la plataforma continental hace $20 \mathrm{ka}$ (Huybrechts, 1993; Ó Cofaigh et al. 2014). A partir de este momento la capa de hielo comienza su retirada a posiciones actuales (Ingólfsson et al. 1998; Ingólfsson, 2004; Ó Cofaigh et al. 2014). La tendencia fue el retroceso continuo, sin embargo, la evidencia paleoclimática señala la ocurrencia de una serie de oscilaciones climáticas que provocan un avance glacial en el Holoceno medio y el período neoglacial (López-Martínez \& Serrano, 2004; Ingólfsson, 2004; Hall, 2009; Fretwell et al. 2010; Watcham et al. 2011; Simms et al. 2012). El comportamiento de la capa de hielo impulsó procesos eustáticos e isostáticos que terminan por exponer y configurar espacialmente áreas libres de hielo (López-Martínez \& Serrano, 2004; Fretwell et al. 2010; Watcham et al. 2011). Éstas corresponden actualmente al 1\% del total de la superficie, principalmente ubicadas en las zonas costeras (Lee et al. 2017). En ellas se concentra gran parte de la biodiversidad vegetal del continente, regulada por la interacción de las variables abióticas que definen los nichos ecológicos (Soberón \& Peterson, 2005; Soberón, 2007; Soberón \& Nakamura, 2009; Peterson, 2009) de las comunidades vegetales. A diferencia de otras regiones del mundo donde la depredación y la competencia tienen impactos sustantivos en las distribuciones de especies, en Antártica son los factores abióticos los determinantes para la distribución de las especies terrestres (Lee et al. 2017). Los principales factores que intervienen en la distribución de las formaciones vegetales antárticas son luminosidad, radiación solar, temperatura, viento, precipitación, disponibilidad hídrica, suelos, dinámica geomorfológica, fauna y proximidad del mar (Cañadas, 2003).

Estos factores se utilizan en la construcción de modelos de distribución potencial con los cuales es posible proyectar geográficamente el espacio ecológico o nichos ecológicos (Phillips et al. 2006; Pearson, 2007; Phillips \& Dudík, 2008; Barve et al. 2011) en distintos momentos temporales, tanto para el presente como para el futuro o el pasado (Premoli et al. 2010; Pliscoff \& FuentesCastillo, 2011). El modelo de máxima entropía (Maxent) es uno de los algoritmos que se ha utilizado ampliamente para modelar espacialmente la distribución geográfica de especies considerando las condiciones ambientales de los sitios de ocurrencia conocidos (Phillips et al. 2006).

En Antártica su uso se ha limitado a modelar hábitat de especies marinas como Pygoscelis adeliae Hombron \& Jacquinot, 1841 (Friedlaender et al. 2011), Lobodon carcinophaga Hombron \& Jacquinot, 1841 (Friedlaender et al. 2011; Nachtsheim et al. 2017), Megaptera novaeangliae Borowosky, 1781 y Balaenoptera bonaerensis Burmeister, 1867 (Friedlaender et al. 2011; Bombosch et al. 2014), Amphipneustes lorioli Koehler, 1901 y Ctenocidaris perrieri Koehler, 1901 (Gutt et al. 2012) para momentos actuales dada la disponibilidad de datos oceanográficos. Por el contrario, aún no existe un desarrollo de investigaciones relacionadas con la distribución potencial de las especies vegetales, tanto en momentos de la actualidad como su análisis en el pasado, dado el vacío de información para Antártica de un set de datos bioclimáticos que sustente la elaboración de modelos de distribución potencial.

Si se integran en un modelo de distribución potencial las variables abióticas del nicho actual de Sanionia spp. con las variables abióticas reconstruidas para momentos del Holoceno y sus respectivas áreas libres de hielo para península Fildes, es posible reconstruir la distribución potencial para momentos temporales pasados. 
Este estudio tiene por objetivo proyectar un modelo de distribución potencial de Sanionia spp. para dos momentos temporales del Holoceno $(6$ ka y $11 \mathrm{ka}$ ) en las áreas libres de hielo disponibles en península Fildes, utilizando variables abióticas en base a la topografía y datos de temperatura, la reconstrucción de las áreas libres de hielo basado en la posición de la capa de hielo, transgresiones marinas y rebote isostático. Esto permite aportar con información para identificar las características biogeográficas de la especie en áreas libres de hielo en Antártica, como también para evaluar la dinámica de las poblaciones de la especie en dicho continente en momentos del pasado cercano.

\section{MATERIALES Y MÉTODOS}

Este trabajo se desarrolló en península Fildes situada en el extremo suroriental de la isla Rey Jorge, al nororiente del archipiélago subantártico marítimo de las Shetland del Sur, $160 \mathrm{~km}$ al norte de península Antártica (Fig. 1). La península es la principal área libre de hielo de isla Rey Jorge de 28 $\mathrm{km} 2$. Para efectos de este estudio se analizaron 27,5 $\mathrm{km} 2$. Tiene una extension de $8 \mathrm{~km}$ y su extensión longitudinal varía entre $1,5 \mathrm{~km}$ a $4 \mathrm{~km}$. El área libre de hielo de península Fildes posee evidencias morfológicas que dan cuenta de la ocurrencia de procesos paraglaciares y periglaciares, los cuales comienzan hace $12 \mathrm{ka}$ antes del presente, con el retiro de la capa de hielo y posterior ocurrencia de procesos eustáticos e isostáticos, configurando la actual área libre de hielo de península Fildes. El nivel relativo del mar alcanzó los $15 \mathrm{~m}$ a $18 \mathrm{~m}$ sobre el actual (Wisz et al. 2008; Watcham et al. 2011).

La vegetación criptogámica domina península Fildes (Gimingham \& Smith, 1970) con aproximadamente 109 especies de líquenes y de 40 especies de briofitas (Casanova-Katny et al. 2016). El total de superficie ocupada por la vegetación es de $6,4 \mathrm{~km}^{2}$, de los cuales 5,7 $\mathrm{km}^{2}$ son cobertura de líquenes y un $0,7 \mathrm{~km}^{2}$ es
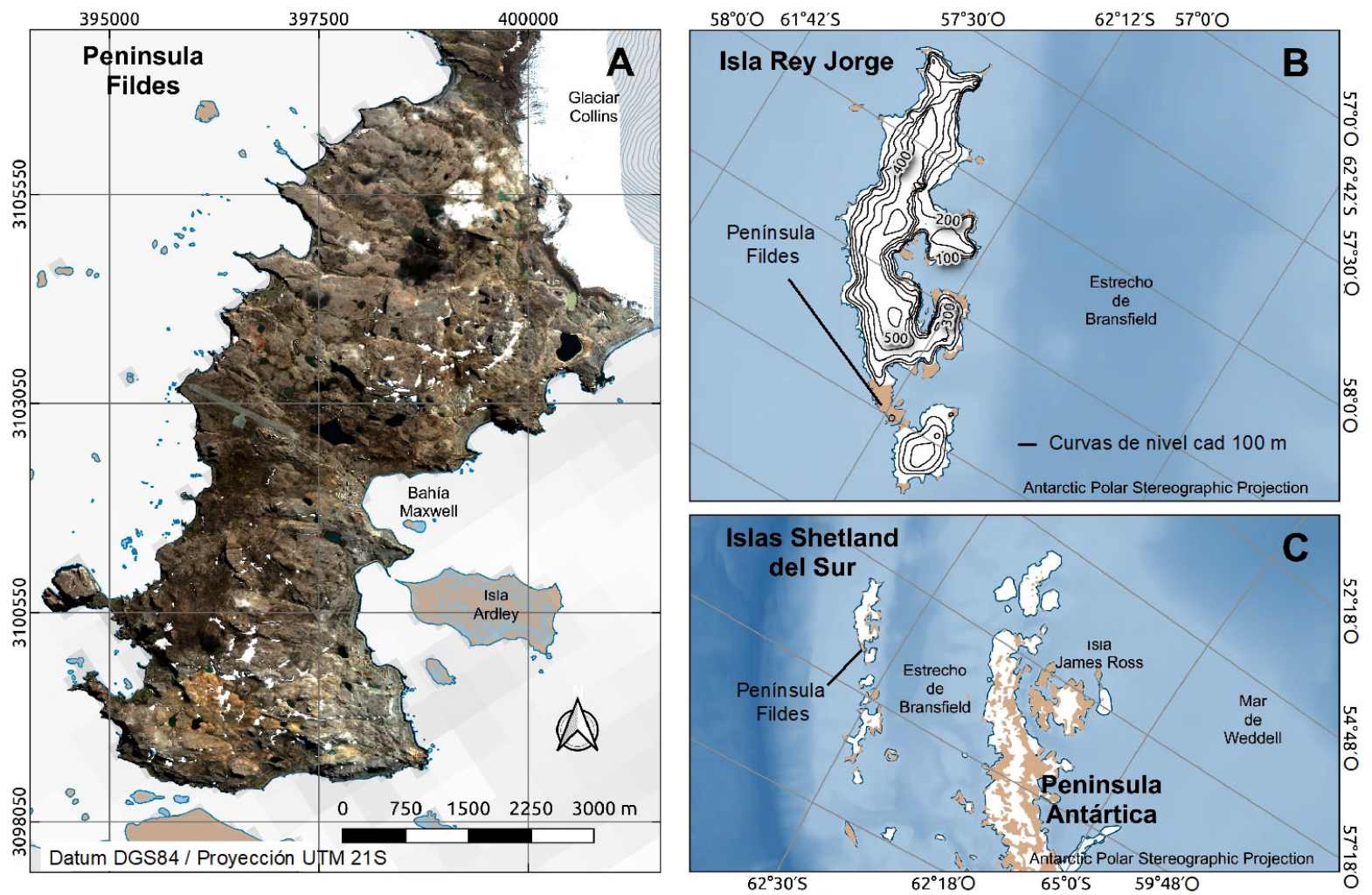

Fig. 1. Localización del área de estudio. A) Península Fildes imagen satelital QuickBird 2010, B) Isla Rey Jorge, en café las áreas libres de hielo y curvas de nivel cada $100 \mathrm{~m}$, C) Isla Rey Jorge en el contexto del archipiélago de las Shetland del Sur y norte de península Antártica, en café áreas libres de hielo. 
superficie ocupada por musgos (Andrade et al. 2018). Esta vegetación junto con las dos únicas plantas vasculares se distribuye dentro de 6 pisos vegetacionales (Cañadas, 2003).

\section{Delimitación del espacio temporal del área libre de hielo}

El área libre de hielo para el momento temporal actual se delimitó en base a cartografía digital oficial (INACH-IGM, 2006) y una imagen satelital QuickBird del año 2010 con resolución $2.4 \mathrm{~m}$ y obtenida el 26 de febrero de 2010 (Fig. 1A). QGIS (QGIS Development Team, 2016) y ArcGIS 10.1 ß fueron utilizadas para digitalizar estos datos en un sistema de coordenadas UTM zona 21. La superficie del área de estudio es representada en el modelo de elevación de terreno (MET Fildes) Presente (pixel $40 \mathrm{~m}$ ), obtenido a través del procesamiento de las curvas de nivel de la cartografía IGM (2006), las cuales están cada 10 $\mathrm{m}$. Las curvas de nivel que hacen referencia en la Fig. 1 no son las que aqui se describen.

La disposición de las áreas libres de hielo en los dos períodos de tiempo pasados $11 \mathrm{ka} \mathrm{y} 6 \mathrm{ka}$ considerados en este trabajo estuvo controlada por las condiciones de extensión del casquete de hielo y altura del nivel medio del mar imperantes. En su estimación se integraron los datos de extensión y espesor del hielo, obtenidos de reconstrucciones basadas en evidencias geológicas y geomorfológicas, terrestres y marinas (Ó Cofaigh et al. 2014) y la reconstrucción del nivel relativo del mar realizada para esas fechas (Watcham et al. 2011). Así, la superficie del terreno usada para proyectar al pasado la distribución potencial de Sanionia spp. corresponde a la superficie actual excluyendo las zonas cubiertas de hielo y que están por debajo del nivel relativo del mar en los momentos temporales pasados, con lo cual se obtuvo los productos MET Fildes 11 ka y MET Fildes 6 ka.

\section{Modelo de distribución \\ potencial de Sanionia spp.}

Para predecir la distribución potencial actual del género Sanionia, se utilizó la versión 3.3.3e del software Maxent (Phillips et al. 2006), que aplica un enfoque de máxima entropía (Maxent) para hacer converger la máxima probabilidad que una especie ocupe un territorio. Las 109 coordenadas de presencia utilizadas para alimentar el modelo de distribución se obtuvieron de colectas de individuos del género Sanionia en península Fildes, en los años 2010 y 2011. Este software utiliza datos de entrenamiento y de validación, por lo que se asumió que un $75 \%$ de los datos de presencia fueron utilizados como puntos de entrenamiento y un $25 \%$ como puntos de validación.

Los datos de variables de entrada asociadas a la topografía y al clima imperantes en las fechas de las corridas del modelo, fueron derivados a partir de la geometría local de cada superficie terrestre base compilada y desde el cálculo de la variación altitudinal de la temperatura.

Para el período actual se generaron archivos raster (249 filas, 199 columnas, $40 \mathrm{~m}$ de tamaño de celda) para calcular valores locales de elevación, pendiente y exposición, a partir del análisis espacial del MET Fildes presente usando extensión 3d análisis de ArcGIS 10.1 ®. La distribución espacial de la temperatura del presente se construyó a partir de un valor de $-2,4{ }^{\circ} \mathrm{C}$ para la temperatura media anual en Fildes y de un gradiente altitudinal de $-0,00725^{\circ} \mathrm{C} \mathrm{m}-1$ (Braun et al. 2004). Consecuentemente, se generó una capa de temperatura local para cada punto de la grilla calculando la ecuación siguiente:

\section{temp present ${ }^{\circ} \mathrm{C}=\left(-2,4-0,00725^{*} \text { hiMET }\right)^{\circ} \mathrm{C}$}

Para la parametrización de la ejecución del modelo Maxent en el presente se definió un límite superior de 500 iteraciones. Para estimar la capacidad de predicción del modelo se analizó el área bajo la curva (AUC), salida gráfica del modelo que representa la capacidad de discriminación de una ausencia o especificidad (Phillips \& Dudík, 2008). Para establecer la probabilidad de presencia se utilizó el formato de salida "logística", cuyo rango va de 0-1. El análisis para determinar la contribución relativa de cada variable se realizó con la prueba de Jacknife (Phillips et al. 2006).

$\mathrm{Al}$ no estar disponible para Antártica el set de datos bioclimáticos de Worldclim (Fick \& Hijmans, 2017) que sustente la elaboración de modelos de distribución potencial se construyeron variables abióticas a partir de la topografía y los 
datos de temperatura disponibles. También fue necesario reconstruir las áreas libres de hielo para los distintos momentos temporales analizados, considerando la posición de la capa de hielo, transgresiones marinas y rebote isostático.

Para el pasado se calcularon los valores locales de elevación, pendiente y exposición para los momentos 11 ka y 6 ka. Se aplicó la herramienta 3d Analyst de ArcGIS 10.1 ® sobre las superficies MET Fildes 11 ka y MET Fildes $6 \mathrm{ka}$, generando archivos raster (249 filas, 199 columnas, $40 \mathrm{~m}$ de tamaño de celda) con los respectivos valores, obteniendo un archivo para cada una de las variables abióticas para el modelo de distribución potencial.

El valor de temperatura promedio anual en superficie fue corregido por la anomalía de temperatura respecto al presente obtenida del análisis isotópico del testigo de hielo de la isla James Ross (Mulvaney et al. 2012), equivalente a $0,780{ }^{\circ} \mathrm{C}$ en período 11 ka y $0,159{ }^{\circ} \mathrm{C}$ para el período $6 \mathrm{ka}$.

temp $\quad 11 \quad \mathrm{ka} \quad{ }^{\circ} \mathrm{C}=\left(\left(-2,4^{\circ} \mathrm{C}+0.780^{\circ} \mathrm{C}\right)+\right.$ $\left.\left(-0,00725^{\circ} \mathrm{C}\right)\right)^{*} \operatorname{hiMET}^{\circ} \mathrm{C}$

temp $6 \mathrm{ka}^{\circ} \mathrm{C}=\left(\left(-2,4^{\circ} \mathrm{C}+0,159^{\circ} \mathrm{C}\right)+\left(-0,00725^{\circ} \mathrm{C}\right)\right)$ *hiMET ${ }^{\circ} \mathrm{C}$

La salida logística resultante del modelo de distribución potencial de Maxent fue reclasificada y procesada en ArcGIS $10.1{ }^{\circledR}$. Se estableció la relación entre los rangos de valores de cada una de las variables construidas y la probabilidad de presencia Sanionia spp. para península Fildes, resultante de la corrida del modelo en Maxent. Luego, se reclasificaron en 5 clases de probabilidades: Clase $0=$ no data, clase $1=0-50$, clase $2=51-60$, clase $3=61-70$ y clase $4=71-87$. El umbral de decisión es el $50 \%$ de probabilidad, definiendo que por sobre este valor el modelo predice por sobre el azar.

Proyección del modelo para el pasado

Se proyectó con Maxent el modelo de distribución potencial para los momentos temporales de $11 \mathrm{ka} \mathrm{y} 6 \mathrm{ka}$, utilizando las variables reconstruidas para el pasado, los parámetros y variables de la calibración del modelo para el momento actual. El formato de salida del modelo evaluado para establecer la probabilidad de presencia es la logística cuyo rango va de 0-1.

El modelo de distribución proyectada para las edades pasadas (11 ka y 6 ka), se basó en dos supuestos. 1.- Al utilizar los datos temperatura del análisis de isótopos de deuterio del testigo de hielo de James Ross (Mulvaney et al. 2012), se asumió que el comportamiento climático en el pasado en la isla James Ross (al oriente de península Antártica) es igual a península Fildes (occidente de península Antártica). 2.- La topografía del pasado (MET Fildes $11 \mathrm{ka}$ y MET Fildes $6 \mathrm{ka}$ ) en península Fildes era igual a la actual, pues solo se modificó su geometría (línea de costa) y altura (cotas).

\section{RESULTADOS}

\section{Reconstrucción área libre de hielo}

Los resultados muestran que el área $\mathrm{A}$ (Fig. 2) estaba descubierta de hielo al menos hace $11 \mathrm{ka}$. Su superficie es de $8,2 \mathrm{~km}^{2}, 29,8 \%$ de la superficie libre de hielo actual analizada. El nivel relativo del mar es de $18 \mathrm{~m}$ por sobre el nivel medio actual. Las alturas son inferiores a las actuales. El rango de elevación actual va de los 0 a los $147 \mathrm{~m}$. El área B estuvo libre de hielo hace 6 $\mathrm{ka}$, tiene una superficie de $21,5 \mathrm{~km}^{2}$, equivalente a $78 \%$ de la superficie actual. Las playas se situaron por sobre las que hoy se observan en península Fildes. El nivel relativo del mar es de $15 \mathrm{~m}$ por sobre el actual y las alturas se encuentran entre 1 m y $149 \mathrm{~m}$ y son inferiores a las actuales. El área $\mathrm{C}$ es el área libre de hielo actual, corresponde la configuración espacial del área libre de hielo que al año 2010 tiene península Fildes. El rango de elevación del área libre de hielo va entre $0 \mathrm{~m} \mathrm{y}$ $159 \mathrm{~m}$ y la superficie es de $27,5 \mathrm{~km}^{2}$.

Distribución potencial actual de Sanionia spp. para península Fildes

Se obtiene un mapa de probabilidad de presencia de Sanionia spp. (Fig. 3). El valor AUC obtenido del MDP S. Presente es de 0,738, indicando que tiene una capacidad predictiva aceptable según Phillips et al. (2006). 


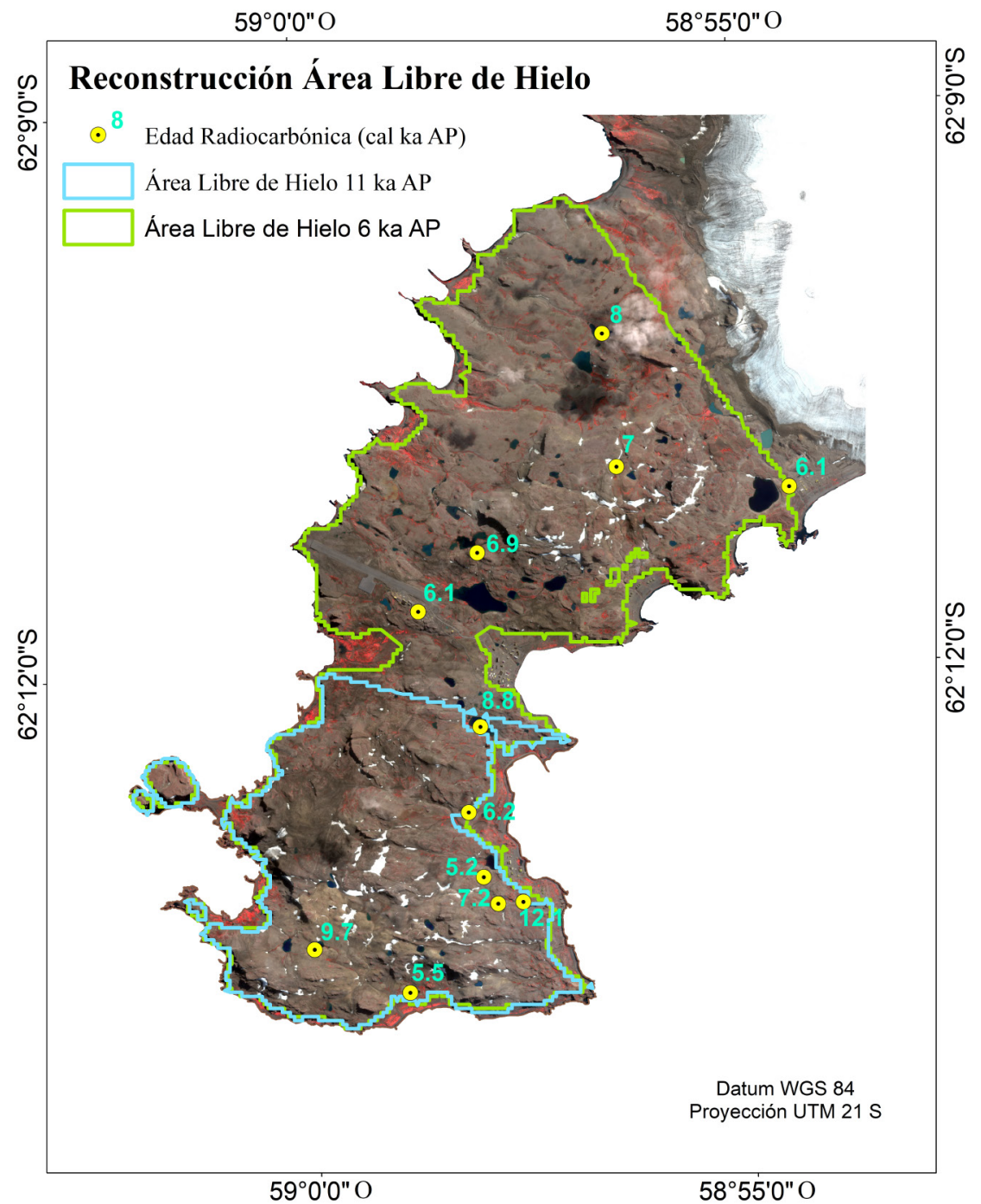

Fig. 2. Áreas libres de hielo. Área A, reconstruida para 11 ka AP y delimitada en línea color verde; Área B, reconstruida para 6 ka AP y delimitada en línea color celeste; Área C, Actual, imagen satelital. Reconstrucción de áreas considerando edades radiocarbónicas compiladas por Ó Cofaigh et al. (2014) y el nivel relativo del mar descrito por Watcham et al. (2011).

Los valores de probabilidad van de $1 \%$ a $87 \%$, siendo este último el valor máximo de probabilidad de presencia. De un total de $27,5 \mathrm{~km}^{2}$ evaluados en península Fildes, $8,1 \mathrm{~km}^{2}$ presentan probabilidad de presencia de Sanionia spp. correspondiente al $29,5 \%$ de la superficie libre de hielo actual. El 20\% de la superficie de península Fildes tiene valores de probabilidad entre el $51 \%$ y $60 \%$, el $6,9 \%$ de la superficie está entre el $61 \%$ y $70 \%$ de probabilidad de presencia y el $22,5 \%$ de la superficie tiene los valores de probabilidades más altas, entre $71 \%$ y $87 \%$.
De las variables utilizadas, la temperatura y la elevación son las que mayor contribución hacen en la predicción de distribución. Porcentualmente ambas variables aportan un $74,5 \%$ en la predicción de distribución potencial de Sanionia ssp. (temperatura 37,6\% y elevación 36,9\%). Por otra parte, el aporte de la exposición $(15,2 \%)$ y la pendiente $(10,3 \%)$ son más bajos.

Existe una relación entre rangos de valores de cada una de las variables y la probabilidad de presencia de Sanionia spp. para península Fildes. La probabilidad 

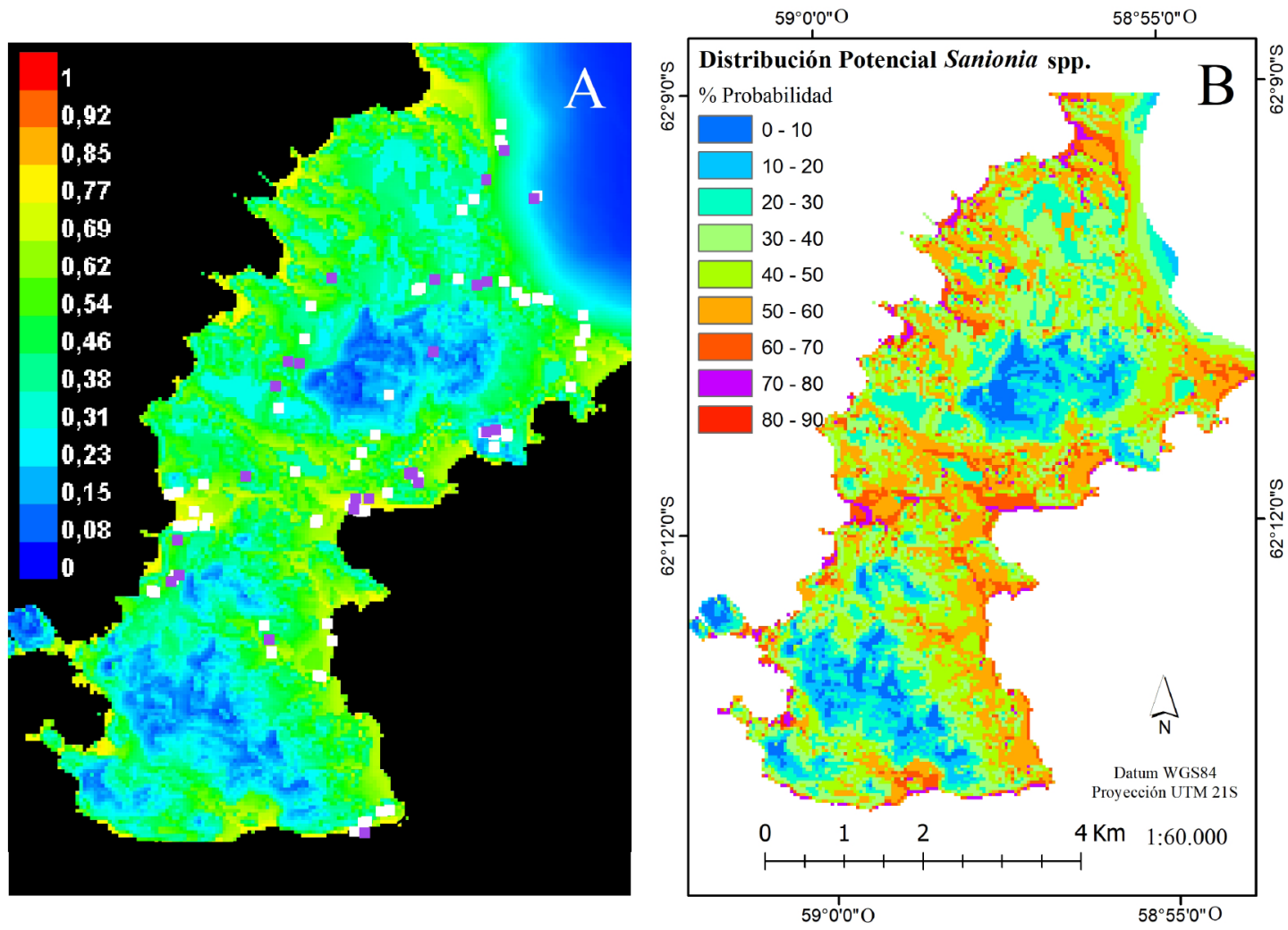

Fig. 3. Distribución potencial de Sanionia spp. La imagen A, es la salida de Maxent en formato logístico. Los valores van de 0 a 1 siendo este valor el máximo de probabilidad de presencia. El umbral de decisión es 0.5. Los puntos blancos son los datos de entrenamiento del modelo y los puntos de color púrpura indican los puntos de validación del modelo. La imagen $\mathrm{B}$ corresponde a valores de probabilidad de presencia del área de análisis reclasificados y expresados en porcentaje de probabilidad (\%).

de presencia aumenta conforme las temperaturas son más cálidas. Las zonas con temperaturas por debajo de los $-3,0^{\circ} \mathrm{C}$ tienen probabilidad inferior al $50 \%$. La probabilidad sobre el $70 \%$ se obtiene con temperaturas minimas de $-2,47^{\circ} \mathrm{C}$. En contraparte la probabilidad de presencia aumenta con valores bajos de elevación y de pendiente. Sobre los $130 \mathrm{~m}$ y con pendientes por encima del $52 \%$ la probabilidad de presencia es por debajo del $50 \%$. Con alturas bajo los 30 m y pendientes de hasta $11 \%$ la probabilidad de presencia es sobre el $60 \%$. En todas las exposiciones es posible encontrar probabilidad de presencia, pero entre los $157^{\circ}$ y los $360^{\circ}$ de exposición la probabilidad es sobre el $70 \%$.

Proyección de la distribución potencial para el pasado

Se logró proyectar para el 11ka y 6 ka el MDP $S$. Presente definiendo espacialmente probabilidad de presencia del género Sanionia para esos momentos pasados. Las áreas libres de hielo con probabilidad de presencia analizadas son superiores para los momentos temporales pasados (Fig. 4).

El área descubierta de hielo hace $11 \mathrm{ka}$ posee una superficie de $8,2 \mathrm{~km}^{2}$ libre de hielo. El área con probabilidad de presencia es de $5,7 \mathrm{~km}^{2}$, correspondiendo al 69,9\% del total de superficie. El área libre de hielo descubierto hace $6 \mathrm{ka}$ tiene una superficie de $21,5 \mathrm{~km}^{2}$, el $58,9 \%$ de esa superficie $\left(12,7 \mathrm{~km}^{2}\right)$ posee probabilidad de presencia de Sanionia spp. (Tabla 1).

\section{DISCUSIÓN Y CONCLUSIÓN}

En los mapas geomorfológicos de LópezMartínez et al. (2012) o Vieira et al. (2015) no se observan arcos morrénicos que permitan asociar con claridad la dinámica del hielo. Es más, las 
Tabla 1. Síntesis valores de superficie y probabilidad.

\begin{tabular}{ccccc}
\hline Edad & $\begin{array}{c}\text { Área } \\
\text { Analizada } \\
\mathrm{Km}^{2}\end{array}$ & $\begin{array}{c}\text { Área Respecto } \\
\text { del total superficie } \\
\text { Península Fildes } \\
\text { Actual } \\
\%\end{array}$ & $\begin{array}{c}\text { Superficie } \\
\text { Probabilidad } \\
\text { Presencia } \\
\mathrm{Km}^{2}\end{array}$ & $\begin{array}{c}\text { \% Superficie } \\
\text { Probabilidad } \\
\text { Presencia }\end{array}$ \\
\hline 11.000 & 8,2 & 29,8 & 5,7 & 69,9 \\
6.000 & 21,5 & 78,3 & 12,7 & 58,9 \\
2010 & 27,5 & 100,0 & 8,1 & 29,5 \\
\hline
\end{tabular}
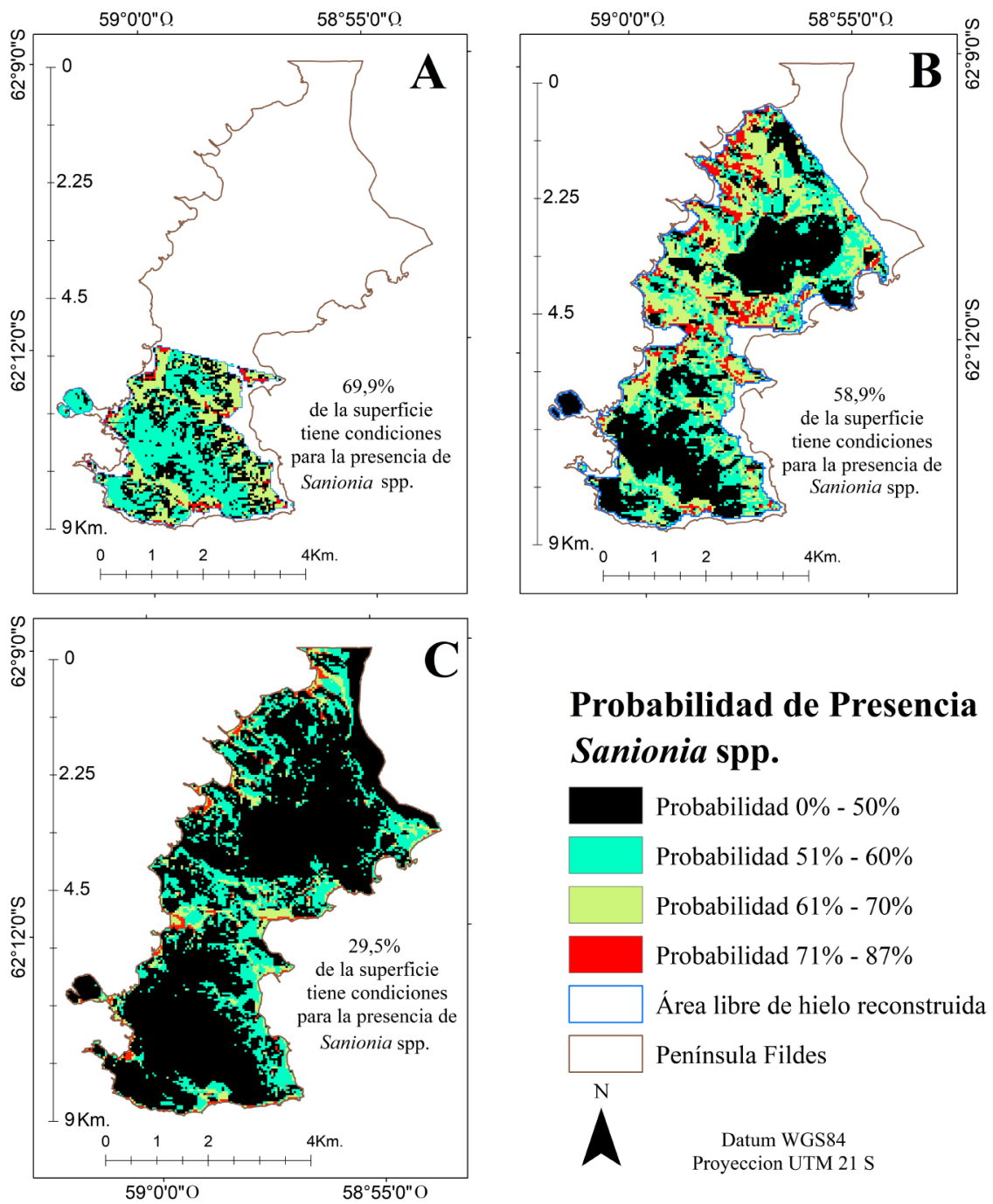

\section{Probabilidad de Presencia Sanionia spp.}

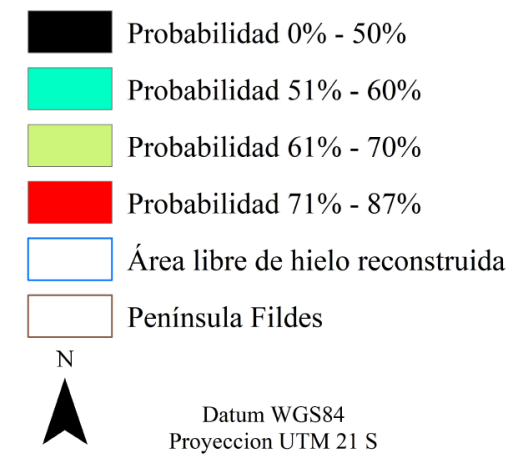

Fig. 4. Probabilidad de presencia de Sanionia spp para tres momentos temporales y sus respectivas áreas libres de hielo. La línea café marca el área libre de hielo actual analizada. La imagen A indica mediante coloración la probabilidad de presencia para el área libre de hielo reconstruida para $11 \mathrm{ka} \mathrm{AP}$. La imagen $\mathrm{B}$ corresponde a la probabilidad de presencia en el área libre de hielo reconstruida para 6 ka AP y la C es la probabilidad de presencia para el año 2010. 
geoformas de origen glacial son anuladas por las formas derivadas de los procesos euto-isostáticos $y$ periglaciares que se han dado desde el último máximo glacial en península Fildes. El trabajo de fotointerpretación de la imagen óptica realizado por los autores no permitió identificar evidencias concluyentes para definir los límites de la capa de hielo. Por lo tanto, integrar en el análisis territorial la localización de edades mínimas de deglaciación (Ó Cofaigh et al. 2014) con modelos glacioeustáticos e isostáticos, surge como un método que permite reconstruir espacialmente áreas libres de hielo en zonas costeras de Antártica.

El estudio permitió construir un método de rasterización de variables ambientales para ser utilizado en modelos de distribución potencial de especies en zonas geográficas que no cuentan con datos bioclimáticos, como es el caso de Antártica. Los modelos de distribución potencial basados en los datos del Worldclim usan principalmente variables climáticas (temperatura y precipitación), siendo las de origen topográficas variables complementarias en la construcción de los modelos de nicho ecológico (Fick \& Hijmans, 2017). El MDP S. Presente se sustenta en variables abióticas de origen topográfico siendo un modelo limitado en cuanto a la participación de variables bioclimáticas en la predicción. Sin embargo, el modelo de distribución fue capaz de predecir presencia de manera aceptable según su curva AUC (Phillips et al. 2006), el modelo demostró que teniendo variables topográficas y gradientes altitudinales de condiciones atmosféricas se puede construir el nicho ecológico de especies en áreas libres de hielo de Antártica. El método logra resultados con mayor resolución espacial que con los modelos de distribución potencial construidos con las 19 variables bioclimáticas.

El análisis espacial del MDP $S$. Presente resultante de Maxent permitió definir el rango de valores de cada variable predictiva del modelo acercándose a configurar el nicho ecológico del género Sanionia spp. La variable de temperatura es la única variable que tiene una relación directa con la presencia de Sanionia spp. A mayor temperatura mayor es la probabilidad de presencia, en comparación con las variables de elevación y pendiente cuya relación es inversa. Según el análisis de los resultados del MDP S. Presente las zonas donde existe probabilidad de presencia tienen las condiciones ambientales siguientes: temperaturas entre los $-2,4^{\circ} \mathrm{C}$ y los $-3,0^{\circ} \mathrm{C}$, alturas bajo los 130 $\mathrm{m}$, pendientes bajo los $52 \%$ y exposiciones sur este, sur - oeste y noroeste.

La temperatura es la variable que tiene el mayor peso en la predicción, 37,6\%. Dado que los momentos temporales 11 ka y 6 ka presentan anomalías de temperatura superiores al presente, la probabilidad de presencia de Sanionia spp. aumenta para los estadios temporales pasados. Para el 11 ka el 69,9\% de superficie tiene sobre el $50 \%$ de probabilidad de presencia de Sanionia spp., siendo la anomalía de temperatura en ese momento de $0,78^{\circ} \mathrm{C}$ por sobre el promedio actual. La anomalía de temperatura para el momento temporal de $6 \mathrm{ka}$ está por sobre el promedio actual en $0,159^{\circ} \mathrm{C}$ y la superficie con probabilidad de presencia es de 58,9\%. El momento actual, a pesar de tener una mayor superficie libre de hielo la superficie con probabilidad de presencia de Sanionia spp. es de $29,5 \%$, debido a que la temperatura es menor que la que había en los momentos temporales pasados evaluados.

Este estudio proporcionó un método que permitió la construcción de un modelo de distribución potencial de Sanionia spp, aplicable tanto para otras especies nativas e introducidas de la región como para distintos estadios temporales $y$ en zonas donde no existen datos bioclimáticos espacializados. Finalmente este trabajo aporta a la hipótesis de distribución de especies, por ejemplo en la disciplina de la genética de poblaciones nativas y puede sustentar la elaboración de herramientas de planificación para la conservación de zonas vulnerables como son las áreas libres de hielo en Antártica.

\section{AGRADECIMIENTOS}

Los autores agradecen al proyecto INACH T_17-09.

\section{LITERATURA CITADA}

Andrade, A. M. D., Michel, R. F. M., Bremer, U. F., Schaefer, C. E. G. R., \& Simões, J. C. (2018). Relationship between solar radiation 
and surface distribution of vegetation in Fildes Peninsula and Ardley Island, Maritime Antarctica. International Journal of Remote Sensing, 39(8), 2238-2254.

Barve, N., Barve, V., Jiménez-Valverde, A., LiraNoriega, A., Maher, S. P., Peterson, A. T., ... \& Villalobos, F. (2011). The crucial role of the accessible area in ecological niche modeling and species distribution modeling. Ecological Modelling, 222(11), 18101819.

Bombosch, A., Zitterbart, D. P., Van Opzeeland, I., Frickenhaus, S., Burkhardt, E., Wisz, M. S., \& Boebel, O. (2014). Predictive habitat modelling of humpback (Megaptera novaeangliae) and Antarctic minke (Balaenoptera bonaerensis) whales in the Southern Ocean as a planning tool for seismic surveys. Deep Sea Research Part I: Oceanographic Research Papers, 91, 101114.

Braun, M., Saurer, H., \& Gobmann, H. (2004). Climate, energy fluxes and ablation rates on the ice cap of King George Island. Pesquisa Antártica Brasileira, 4, 87-103.

Cañadas, E. S. (2003). Paisaje natural y pisos geoecológicos en las áreas libres de hielo de la Antártida marítima: Islas Shetland del Sur. Boletín de la Asociación de Geógrafos Españoles, (35), 5-32.

Casanova-Katny, A., Torres-Mellado, G. A., \& Eppley, S. M. (2016). Reproductive output of mosses under experimental warming on Fildes Peninsula, King George Island, maritime Antarctica. Revista Chilena de Historia Natural, 89(1), 1-9.

Fick, S. E., \& Hijmans, R. J. (2017). WorldClim 2: New 1-km spatial resolution climate surfaces for global land areas. International Journal of Climatology, 37(12), 4302-4315.

Fretwell, P. T., Hodgson, D. A., Watcham, E. P., Bentley, M. J., \& Roberts, S. J. (2010). Holocene isostatic uplift of the South Shetland Islands, Antarctic Peninsula, modelled from raised beaches. Quaternary Science Reviews, 29(15), 1880-1893.

Friedlaender, A. S., Johnston, D. W., Fraser, W. R., Burns, J., Patrick, N. H., \& Costa, D. P. (2011). Ecological niche modeling of sympatric krill predators around Marguerite Bay, Western Antarctic Peninsula. Deep Sea Research Part II: Topical Studies in Oceanography, 58(13), 1729-1740.

Gimingham, C. H., \& Smith, R. I. L. (1970). Bryophyte and lichen communities in the maritime Antarctic. Holdgate, M. W. Antarctic Ecology, 2, 752-785.

Gutt, J., Zurell, D., Bracegridle, T., Cheung, W., Clark, M., Convey, P., ... \& Grimm, V. (2012). Correlative and dynamic species distribution modelling for ecological predictions in the Antarctic: A crossdisciplinary concept. Polar Research, 31(1).

Hall, B. L. (2009). Holocene glacial history of Antarctica and the sub-Antarctic islands. Quaternary Science Reviews, 28(21), 2213-2230.

Huybrechts, P. (1993). Glaciological Modelling of the Late Cenozoic East Antarctic Ice Sheet: Stability or Dynamism? Geografiska Annaler: Series A, Physical Geography, 75(4), 221-238.

INACH-IGM. (2006). República de Chile: Isla Rey Jorge-Península Fildes / XII Región de Magallanes y de la Antártica Chilena. Escala 1:10.000. Santiago de Chile. Instituto Antártico Chileno/Instituto Geográfico Militar.

Ingólfsson, Ó. (2004). Quaternary glacial and climate history of Antarctica. En J. Ehlers \& P. L. Gibbard (Eds.), Developments in Quaternary Sciences, 2, 3-43.

Ingólfsson, Ó., Hjort, C., Berkman, P. A., Björck, S., Colhoun, E., Goodwin, I. D., ... \& Prentice, M. L. (1998). Antarctic glacial history since the Last Glacial Maximum: An overview of the record on land. Antarctic Science, 10(3), 326-344.

Lee, J. R., Raymond, B., Bracegirdle, T. J., Chadès, I., Fuller, R. A., Shaw, J. D., \& Terauds, A. (2017). Climate change drives expansion of Antarctic ice-free habitat. Nature, 547(7661), 49-54.

López-Martínez, J., \& Serrano, E. (2004). Morfogénesis periglaciar y deglaciación en las penínsulas Barton y Weaver (islas Shetland del sur, Antártida). Boletín de la Real Sociedad Española de Historia Natural. Sección 
geológica, 99(1), 131-140.

López-Martínez, J., Serrano, E., Schmid, T., Mink, S., \& Linés, C. (2012). Periglacial processes and landforms in the South Shetland Islands (northern Antarctic Peninsula region). Geomorphology, 155-156, 62-79.

Mulvaney, R., Abram, N. J., Hindmarsh, R. C. A., Arrowsmith, C., Fleet, L., Triest, J., ... \& Foord, S. (2012). Recent Antarctic Peninsula warming relative to Holocene climate and ice-shelf history. Nature, 489(7414), 141144.

Nachtsheim, D. A., Jerosch, K., Hagen, W., Plötz, J., \& Bornemann, H. (2017). Habitat modelling of crabeater seals (Lobodon carcinophaga) in the Weddell Sea using the multivariate approach Maxent. Polar Biology, 40(5), 961-976.

Ó Cofaigh, C., Davies, B. J., Livingstone, S. J., Smith, J. A., Johnson, J. S., Hocking, E. P., ... \& Simms, A. R. (2014). Reconstruction of ice-sheet changes in the Antarctic Peninsula since the Last Glacial Maximum. Quaternary Science Reviews, 100, 87-110.

Pearson, R. G. (2007). Species' Distribution Modeling for Conservation Educators and Practitioners. Lesson in Conservation, 3, 54-89.

Peterson, A. T. (2009). Phylogeography is not enough: The need for multiple lines of evidence. Frontiers of Biogeography, 1(1), 1-8.

Phillips, S. J., \& Dudík, M. (2008). Modeling of species distributions with Maxent: New extensions and a comprehensive evaluation. Ecography, 31(2), 161-175.

Phillips, S. J., Anderson, R. P., \& Schapire, R. E. (2006). Maximum entropy modeling of species geographic distributions. Ecological Modelling, 190(3), 231-259.

Pliscoff, P., \& Fuentes-Castillo, T. (2011). Modelación de la distribución de especies y ecosistemas en el tiempo y en el espacio: Una revisión de las nuevas herramientas y enfoques disponibles. Revista de geografía Norte Grande, (48), 61-79.

Premoli, A. C., Mathiasen, P., \& Kitzberger,
T. (2010). Southern-most Nothofagus trees enduring ice ages: Genetic evidence and ecological niche retrodiction reveal high latitude $\left(54^{\circ} \mathrm{S}\right)$ glacial refugia. Palaeogeography, Palaeoclimatology, Palaeoecology, 298(3), 247-256.

QGIS Development Team (2016). QGIS Geographic Information System. Open Source Geospatial Foundation Project. http://qgis.osgeo.org

Simms, A. R., Ivins, E. R., DeWitt, R., Kouremenos, P., \& Simkins, L. M. (2012). Timing of the most recent Neoglacial advance and retreat in the South Shetland Islands, Antarctic Peninsula: Insights from raised beaches and Holocene uplift rates. Quaternary Science Reviews, 47, 41-55.

Soberón, J. (2007). Grinnellian and Eltonian niches and geographic distributions of species. Ecology Letters, 10(12), 1115-1123.

Soberón, J., \& Peterson, A. T. (2005). Interpretation of Models of Fundamental Ecological Niches and Species' Distributional Areas. Biodiversity Informatics, 2, 1-10.

Soberón, J., \& Nakamura, M. (2009). Niches and distributional areas: Concepts, methods, and assumptions. Proceedings of the National Academy of Sciences, 106 (Supplement 2), 19644-19650.

Vieira, R., Marotta, H., Rosa, K. K. da, Jaña, R., Simões, C. L., Júnior, E. de S., ... \& Felizardo, J. P. de S. (2015). Análisis Sedimentológico y geomorfológico de áreas lacustres en la Península Fildes, Isla Rey Jorge, Antártica Marítima. Investigaciones Geográficas, (49), 3-30.

Watcham, E. P., Bentley, M. J., Hodgson, D. A., Roberts, S. J., Fretwell, P. T., Lloyd, J. M., ... \& Moreton, S. G. (2011). A new Holocene relative sea level curve for the South Shetland Islands, Antarctica. Quaternary Science Reviews, 30(21), 3152-3170.

Wisz, M. S., Hijmans, R. J., Li, J., Peterson, A. T., Graham, C. H., \& Guisan, A. (2008). Effects of sample size on the performance of species distribution models. Diversity and Distributions, 14(5), 763-773. 
